\title{
ON THE PRODUCT OF LEGENDRE AND BESSEL FUNCTIONS
}

F. M. RAGAB

1. Introduction. The following formulae will be established: If $|\operatorname{amp} z|<3 \pi / 2, R(1-m)>0$,

$$
\begin{aligned}
& \int_{0}^{\infty} \lambda^{-m / 2}(1+\lambda)^{(m-\nu) / 2} K_{\nu}\left(z(1+\lambda)^{1 / 2}\right) P_{n}^{m}(1+2 \lambda) d \lambda \\
& \quad=\frac{z^{\nu-2 m-2}}{2^{\nu-2 m} \cdot \pi} \sum_{i,-i} \frac{1}{i} E\left(1+n+m, m-n, m-\nu+1:: e^{i \pi} \cdot z^{2} / 4\right)
\end{aligned}
$$

where $\sum_{2,-i}$ denotes that to the expression following it a similar expression with $i_{1}-i$ interchanged is to be added. If $R(1 / 4+\nu / 2-n)$ $>0, R(5 / 4+\nu / 2+n)>0, R(1-m)>0$ and $z$ is real and positive.

$$
\begin{aligned}
& \int_{0}^{\infty} \lambda^{-m / 2}(1+\lambda)^{m / 2-\nu / 2} J_{\nu}\left(z(1+\lambda)^{1 / 2}\right) P_{n}^{m}(1+2 \lambda) d \lambda \\
& =\frac{\Gamma(2 m+1)}{\Gamma(n-m+1) \Gamma(\nu-n)}\left(\frac{1}{2} z\right)^{\nu-2 n-2} \cdot{ }_{1} F_{2}\left(\begin{array}{l}
m-n ;-z^{2} / 4 \\
\nu-n,-2 n
\end{array}\right) \\
& +\frac{\Gamma(-2 n-1)}{\Gamma(-m-n) \Gamma(1+n+\nu)}\left(\frac{1}{2} z\right)^{2 n+\nu} \cdot{ }_{1} F_{2}\left(\begin{array}{cc}
1+m+n ; & -z^{2} / 4 \\
1+n+\nu, & 2+2 n
\end{array}\right) .
\end{aligned}
$$

These two formulae will be deduced from more general formulae [(9) and (14) below] by means of the formula

$$
\begin{aligned}
& P_{n}^{m}(1+2 \lambda) \\
& \quad=\frac{1}{\Gamma(1-m)}\{\lambda(1+\lambda)\}^{-m / 2} F\left(\begin{array}{c}
-m-n, n-m+1 ;-\lambda \\
1-m
\end{array}\right) .
\end{aligned}
$$

For the definition of the $E$-functions, the reader is referred to (T. M. Macrobert, Functions of a complex variable, 4th ed., Glasgow, 1954, p. 352). Formula (1) will be proved in $\$ 3$ by means of the following subsidiary formula

$$
\frac{2 \pi z^{\alpha+\beta-1} e^{-z}}{\Gamma(1-\alpha) \Gamma(1-\beta)} E(1-\alpha, 1-\beta:: z)=\sum_{i,-i} \frac{1}{i} E\left(\alpha, \beta:: e^{i \pi} z\right),
$$

which will be proved in $\S 2$. (2) will be proved in $\$ 4$. The following formulae will be required in the proofs:

Received by the editors April 29, 1957. 


$$
\begin{gathered}
E\left(p ; \alpha_{r}: q ; \rho_{s}: z\right)=\prod_{s=1}^{p} \Gamma\left(\alpha_{s}-\alpha_{r}\right)\left\{\prod_{t=1}^{q} \Gamma\left(\rho_{t}-\alpha_{r}\right)\right\}^{-1} \Gamma\left(\alpha_{r}\right) \\
\times z^{\alpha_{r} F}\left\{\begin{array}{l}
\alpha_{r}, \alpha_{r}-\rho_{1}+1, \cdots, \alpha_{r}-\rho_{q}+1 ;(-1)^{p-q_{z}} z \\
\alpha_{r}-\alpha_{1}+1, \cdots * \cdots, \alpha_{r}-\alpha_{p}+1
\end{array}\right\} ; \\
\int_{0}^{\infty} e^{-\left(t+z^{2} / t\right) / 2 t^{-\nu-1} d t=2 z^{-\nu} K_{\nu}(z),}
\end{gathered}
$$

where $R(z)>0, R\left(z^{2}\right)>0$;

$$
\int_{0}^{\infty} e^{-\lambda z} \lambda^{\gamma-1} F\left(\begin{array}{c}
\alpha, \beta ;-\lambda \\
\gamma
\end{array}\right) d \lambda=\frac{\Gamma(\gamma)}{\Gamma(\alpha) \Gamma(\beta)} z^{-\gamma} E(\alpha, \beta:: z) ;
$$

where $R(\gamma)>0, R(z)>0$; and

$$
\int_{0}^{\infty} e^{-\lambda} \lambda^{\alpha_{p+1}-1} E\left(p ; \alpha_{r}: q ; \rho_{s}: z / \lambda\right) d \lambda=E\left(p+1 ; \alpha_{r}: q ; \rho_{s}: z\right),
$$

where $R\left(\alpha_{p+1}\right)>0$.

These formulae are to be found in the book referred to on pp. 353, 389,393 and 394 respectively.

2. Proof of the subsidiary formula. To prove (4) expand each $E$-function on the right hand side of (4) by means of (5), add similar terms, so getting

$$
\begin{aligned}
E\left(\alpha, \beta:: e^{i \pi} z\right)-E\left(\alpha, \beta:: e^{-i \pi} z\right)=2 \pi i \\
\quad \times\left[\frac{\Gamma(\beta-\alpha)}{\Gamma(1-\alpha)} z^{\alpha} F\left(\begin{array}{c}
\alpha ;-z \\
1+\alpha-\beta
\end{array}\right)+\frac{\Gamma(\alpha-\beta)}{\Gamma(1-\beta)} z^{\beta} F\left(\begin{array}{c}
\beta ;-z \\
1+\beta-\alpha
\end{array}\right)\right] \\
=\frac{(2 \pi i) z^{\alpha+\beta-1}}{\Gamma(1-\alpha) \Gamma(1-\beta)} e^{-z} \cdot \sum_{\alpha, \beta} \Gamma(\alpha-\beta) \Gamma(1-\alpha) z^{1-\alpha} F\left(\begin{array}{c}
1-\alpha ; z \\
1+\beta-\alpha
\end{array}\right),
\end{aligned}
$$

where we have used the well known formula $F(\alpha ; \rho ; z)=e^{z} F(\rho-\alpha ; \rho$; $-z$ ). If we apply (5) again, formula (4) is obtained.

3. A generalization of formula (1). The formula to be proved is

$$
\int_{0}^{\infty} \lambda^{\gamma-1}(1+\lambda)^{-\nu / 2} K_{\nu}\left(z(1+\lambda)^{1 / 2}\right) F(\alpha, \beta ; \gamma ;-\lambda) d \lambda=\Gamma(\gamma)
$$

$$
\begin{aligned}
& \times(z / 2)^{2 \alpha+2 \beta+\nu-2 \gamma-2} \\
& \cdot \frac{1}{4 \pi} \sum_{i,-i} \frac{1}{i} E\left(1-\alpha, 1-\beta, 1+\gamma-\nu-\alpha-\beta:: e^{i \pi} \cdot z^{2} / 4\right)
\end{aligned}
$$


where $R(\gamma)>0$, $|\operatorname{amp} z|<\pi$.

On substituting from (6) on the left hand side of (9) and changing the order of integration it becomes

$$
\frac{1}{2} z^{\nu} \int_{0}^{\infty} e^{-t / 2-z^{2} / 2 t} \cdot t^{-\nu-1} d t \int_{0}^{\infty} e^{-\lambda z^{2} / 2 t} \cdot \lambda^{\gamma-1} F\left(\begin{array}{c}
\alpha, \beta ;-\lambda \\
\gamma
\end{array}\right) d \lambda .
$$

From (7), it follows that the inmost integral is equal to

$$
\Gamma(\gamma)\{\Gamma(\alpha) \Gamma(\beta)\}^{-1}\left(z^{2} / 4 t\right)^{-\gamma} E\left(\alpha, \beta:: z^{2} / 2 t\right) .
$$

Thus on replacing $t$ by $2 t$, the expression reduces to

$$
\frac{\Gamma(\gamma)}{\Gamma(\alpha) \Gamma(\beta)} 2^{2 \gamma-\nu-1} z^{\nu-2 \gamma} \cdot \int_{0}^{\infty} e^{-t-z^{2} / 4 t} t^{\gamma-\nu-1} E\left(\alpha, \beta:: \frac{z^{2}}{4 t}\right) d t .
$$

Here substitute for the last $E$-function from (4); and the expression becomes

$$
\begin{aligned}
& \Gamma(\gamma)(z / 2)^{2 \alpha+2 \beta+\nu-2 \gamma-2} \\
& \quad \times \sum_{i,-i} \frac{1}{4 \pi i} \int_{0}^{\infty} e^{-t} t^{\gamma-\nu-\alpha-\beta} E\left(1-\alpha, 1-\beta:: e^{i \pi} z^{2} / 4 t\right) d t,
\end{aligned}
$$

and from this by applying (8), (9) follows.

Special Cases. In (9), take $\alpha=-m-n, \beta=-m+n+1, \gamma=1-m$, apply (3) and so obtain (1).

Next, by expanding each $E$-function on the right hand side of (9) by means of (5); it can be written in the form

$$
\begin{gathered}
\int_{0}^{\infty} \lambda^{\gamma-1}(1+\lambda)^{-\nu / 2} K_{\nu}\left(z(1+\lambda)^{1 / 2}\right) F(\alpha, \beta ; \gamma ;-\lambda) d \lambda=\frac{\Gamma(\gamma)}{4 \pi} \\
\times \sum_{i,-i} \frac{1}{i} E\left(\begin{array}{c}
-\frac{1}{2} \nu, \alpha-\gamma+\frac{1}{2} \nu, \beta-\gamma+\frac{1}{2} \nu, 1: e^{i \pi} \cdot z^{2} / 4 \\
\alpha+\beta-\gamma+\frac{1}{2} \nu
\end{array}\right)
\end{gathered}
$$

where $R(\gamma)>0$, $|\operatorname{amp} z|<\pi$.

In (10) take $\beta=0, \gamma=\alpha+\nu / 2-1$; then if $R(\alpha+\nu / 2-1)>0$, $R(z)>0$

$$
\begin{aligned}
& \int_{0}^{\infty} \lambda^{\alpha+\nu / 2-2}(1+\lambda)^{-\nu / 2} K_{\nu}\left(z(1+\lambda)^{1 / 2}\right) d \lambda=\Gamma\left(\alpha+\frac{1}{2} \nu-1\right) \\
& \times \sum_{i,-i} \frac{1}{4 \pi i} E\left(1-\alpha, 1,-\frac{1}{2} \nu:: e^{i \pi} \cdot z^{2} / 4\right)
\end{aligned}
$$


But, by expanding the following $E$-functions by means of (5), it can be seen that

$$
\begin{aligned}
\sum_{i,-i} & \frac{1}{4 \pi i} E\left(1-\alpha,-\frac{1}{2} \nu, 1:: e^{i \pi} z^{2} / 4\right)=(z / 2)^{-\alpha-\nu / 2+1} \\
& \times \sum_{i,-i} \frac{1}{4 \pi i} E\left(\frac{1}{2}-\frac{1}{2} \alpha+\frac{1}{4} \nu,-\frac{1}{4} \nu+\frac{1}{2} \alpha-\frac{1}{2}:: \frac{e^{i \pi} z^{2}}{4}\right) \\
& =\left(\frac{z}{2}\right)^{1-\alpha-\nu / 2} K_{1-\alpha+\nu / 2}(z) .
\end{aligned}
$$

In (11) take $\alpha=2+\mu-\nu / 2$ and apply (12), so getting

$$
\int_{0}^{\infty} \frac{K_{\nu}\left(z(1+\lambda)^{1 / 2}\right)}{(1+\lambda)^{\nu / 2}} \lambda^{\mu} d \lambda=\frac{2^{\mu+1}}{z^{\mu+1}} \Gamma(\mu+1) K_{\nu-\mu+1}(z),
$$

where $R(\mu)>-I, R(z)>0$.

This formula was given by G. N. Watson (Theory of bessel functions, Cambridge, 1944 , p. 417 , formula (6)).

4. A generalization of (2). The formula to be proved is

$$
\begin{aligned}
\int_{0}^{\infty} \lambda^{\gamma-1}(1+\lambda)^{-\nu / 2} J_{\nu}\left(z(1+\lambda)^{1 / 2}\right) F(\alpha, \beta ; \gamma ;-\lambda) d \lambda=\Gamma(\gamma) \\
\times \sum_{\alpha, \beta} \frac{\Gamma(\beta-\alpha)}{\Gamma(\beta) \Gamma(1+\alpha-\gamma+\nu)}\left(\frac{z}{2}\right)^{2 \alpha-2 \gamma+\nu} \\
{ }_{1} F_{2}\left(\begin{array}{c}
1-\beta ;-z^{2} / 4 \\
1+\alpha-\gamma+\nu, 1+\alpha-\beta
\end{array}\right)
\end{aligned}
$$

where $R(\gamma)>0, R(5 / 4-\gamma+\nu / 2+\alpha)>0, R(5 / 4-\gamma+\nu / 2+\beta)>0$ and $z$ is real and positive.

Proof of (14). In (10), let it be assumed that $R(\gamma) 0, R(5 / 4-\gamma$ $+\nu / 2+\alpha)>0 R(5 / 4-\gamma+\nu / 2+\beta)>0$ and let amp $z$ decrease by $\pi / 2$, so that $z / i$ is written for $z$, then, since $K_{\nu}(t)=i^{\nu} G_{\nu}(i t)$, we have

$$
\begin{aligned}
& \int_{0}^{\infty} \lambda^{\gamma-1}(1+\lambda)^{-\nu / 2} G_{\nu}\left(z(1+\lambda)^{1 / 2}\right) F(\alpha, \beta ; \gamma ;-\lambda) d \lambda=\frac{\Gamma(\gamma)}{4 \pi i} i^{-\nu} \\
& \times\left\{\begin{array}{l}
E\left(-\frac{1}{2} \nu, \alpha-\gamma+\frac{1}{2} \nu, \beta-\gamma+\frac{1}{2} \nu, 1: \alpha+\beta-\gamma+\frac{1}{2} \nu: z^{2} / 4\right) \\
-E\left(-\frac{1}{2} \nu, \alpha-\gamma+\frac{1}{2} \nu, \beta-\gamma+\frac{1}{2} \nu, 1: \alpha+\beta-\gamma+\frac{1}{2} \nu: e^{-2 i \pi} \cdot z^{2} / 4\right)
\end{array}\right\} .
\end{aligned}
$$

Now expand each $E$-function on the right hand side of the last expres- 
sion by means of (5), add applying the formula

$$
\Gamma(z) \Gamma(1-z)=\pi / \sin \pi z ;
$$

so getting if $R(\gamma)>0$

(16)

$$
\begin{aligned}
& \int_{0}^{\infty} \lambda^{\gamma-1}(1+\lambda)^{-\nu / 2} G_{\nu}\left(z(1+\lambda)^{1 / 2}\right) F(\alpha, \beta ; \gamma ;-\lambda) d \lambda=-\frac{1}{2} \Gamma(\gamma) \\
& \times \frac{\Gamma(\alpha-\gamma+\nu) \Gamma(\beta-\gamma+\nu)}{\Gamma(\alpha+\beta-\gamma+\nu)}\left(\frac{z}{2}\right)^{-\nu} \\
&{ }_{1} F_{2}\left(\begin{array}{c}
-\nu-\alpha-\beta+\gamma+1 ;-z^{2} / 4 \\
1-\nu-\alpha+\gamma, 1-\nu-\beta+\gamma
\end{array}\right)+\frac{1}{2} \Gamma(\gamma) \\
& \times \sum_{\alpha, \beta} \frac{\Gamma(-\nu-\alpha+\gamma) \Gamma(\beta-\alpha)}{\Gamma(\beta)(z / 2)^{2 \gamma-\nu-2 \alpha}} \cdot e^{-i \pi(\alpha-\gamma+\nu)} \\
&{ }_{1} F_{2}\left(\begin{array}{c}
1-\beta ;-z^{2} / 4 \\
1+\alpha-\gamma+\nu, 1+\alpha-\beta
\end{array}\right) .
\end{aligned}
$$

Similarly by increasing amp $z$ by $\pi / 2$ in (10) and proceeding as before, we have if $R(\gamma)>0$

$$
\begin{aligned}
& i^{2 \nu} \int_{0}^{\infty} \lambda^{\gamma-1}(1+\lambda)^{-\nu / 2} G_{\nu}\left(e^{i \pi} z(1+\lambda)^{1 / 2}\right) F(\alpha, \beta ; \gamma ;-\lambda) d \lambda \\
&=- \frac{1}{2} \Gamma(\gamma) \frac{\Gamma(\alpha-\gamma+\nu) \Gamma(\beta-\gamma+\nu)}{\Gamma(\alpha+\beta-\gamma+\nu)}\left(\frac{z}{2}\right)^{-\nu} \\
&{ }_{1} F_{2}\left(\begin{array}{c}
-\nu-\alpha-\beta+\gamma+1 ;-z^{2} / 4 \\
1-\nu-\alpha+\gamma, 1-\nu-\beta+\gamma
\end{array}\right) \\
&+\frac{1}{2} \Gamma(\gamma) \sum_{\alpha, \beta} \frac{\Gamma(-\nu-\alpha+\gamma) \Gamma(\beta-\alpha)}{\Gamma(\beta)(z / 2)^{2 \gamma-\nu-2 \alpha}} e^{i \pi(\alpha-\gamma+\nu)} \\
&{ }_{1} F_{2}\left(\begin{array}{c}
1-\beta ;-z^{2} / 4 \\
1+\alpha-\gamma+\nu, 1+\alpha-\beta
\end{array}\right) .
\end{aligned}
$$

Hence, on applying the formula

$$
\pi i J_{\nu}(t)=G_{\nu}(t)-i^{2 \nu} G_{\nu}\left(e^{i \pi} t\right),
$$

and (15); formula (14) is obtained.

PARTICUlaR CASEs. In (14), take $\alpha=-m-n, \beta=-m+n+1$, $\gamma=1-m$ and apply (3), so getting formula (2). A particular case of interest is obtained by putting $\alpha=0$ in (14). Thus we have if $R(\gamma)>0$, $R(\nu / 2-1 / 4)>0, R(z)>0$, 


$$
\int_{0}^{\infty} \frac{J_{\nu}\left(z(1+\lambda)^{1 / 2}\right)}{(1+\lambda)^{\nu / 2}} \lambda^{\gamma-1} d \lambda=\Gamma(\gamma)(2 / z)^{\gamma} J_{\nu-\gamma}(z),
$$

which is again in Watson's book p. 417, formula (5).

Institute for Advanced Study and

Ein Shams University, Cairo, Egypt 